\title{
Integrin $\alpha 6 \beta 4$ identifies an adult distal lung epithelial population with regenerative potential in mice
}

\author{
Harold A. Chapman, ${ }^{1}$ Xiaopeng Li, ${ }^{1}$ Jonathan P. Alexander, ${ }^{1}$ Alexis Brumwell, ${ }^{1}$ Walter Lorizio, ${ }^{1}$ \\ Kevin Tan, ${ }^{1}$ Arnoud Sonnenberg, ${ }^{2}$ Ying Wei, ${ }^{1}$ and Thiennu H. Vu${ }^{1}$
}

1Department of Medicine, Cardiovascular Research Institute, and Lung Biology Center, UCSF, San Francisco, California, USA.

2University of Copenhagen, Copenhagen, Denmark.

\begin{abstract}
Laminins and their integrin receptors are implicated in epithelial cell differentiation and progenitor cell maintenance. We report here that a previously unrecognized subpopulation of mouse alveolar epithelial cells (AECs) expressing the laminin receptor $\alpha 6 \beta 4$, but little or no pro-surfactant C (pro-SPC), is endowed with regenerative potential. Ex vivo, this subpopulation expanded clonally as progenitors but also differentiated toward mature cell types. Integrin $\beta 4$ itself was not required for AEC proliferation or differentiation. An in vivo embryonic lung organoid assay, which we believe to be novel, was used to show that purified $\beta 4^{+}$adult AECs admixed with E14.5 lung single-cell suspensions and implanted under kidney capsules self-organized into distinct Clara cell $10-\mathrm{kDa}$ secretory protein $\left(\mathrm{CC}^{+}{ }^{+}\right)$airway-like and $\mathrm{SPC}^{+}$saccular structures within 6 days. Using a bleomycin model of lung injury and an SPC-driven inducible cre to fate-map AECs, we found the majority of type II AECs in fibrotic areas were not derived from preexisting type II AECs, demonstrating that $\mathrm{SPC}^{-}$progenitor cells replenished type II AECs during repair. Our findings support the idea that there is a stable AEC progenitor population in the adult lung, provide in vivo evidence of AEC progenitor cell differentiation after parenchymal injury, and identify a strong candidate progenitor cell for maintenance of type II AECs during lung repair.
\end{abstract}

\section{Introduction}

Basement membrane laminins and their integrin receptors are critical to lung development and implicated in epithelial cell differentiation and progenitor cell maintenance (1-3). There are 3 major integrin laminin receptors, all of which are expressed in the lung and primarily in epithelial cells: $\alpha 3 \beta 1, \alpha 6 \beta 1$, and $\alpha 6 \beta 4$ (4). $\alpha 6 \beta 4$ is thought to be particularly important in epithelial cell adhesion to basement membranes because this integrin has a uniquely long cytoplasmic tail that promotes assembly of $\alpha 6 \beta 4$ into hemidesmosomes (5). In humans, mutations of either $\alpha 6$ or $\beta 4$, which only partners with $\alpha 6$, are known to result in varying degrees of a blistering skin phenotype depending on the degree of loss of integrin function (6). Skin blistering and sloughing of mucosal epithelial cells have also been reported in integrin $\beta 4$-deficient mice (7). To define the role of this integrin in lung homeostasis, we generated mice with epithelial-specific deletion of integrin $\beta 4$ and characterized the resulting effect on lung function. Unexpectedly, these mice appeared normal and had a normal lifespan. Although $\alpha 6 \beta 4$ is thought to be primarily localized to conducting airways of the lung, in the course of this work we discovered that a substantial fraction of distal lung/alveolar epithelial cells (AECs) expressing little or none of the canonical Clara cell $10-\mathrm{kDa}$ secretory protein (CC10) or pro-surfactant protein C (pro-SPC) also expressed $\alpha 6 \beta 4$. These cells were found to clonally expand ex vivo and to be capable of multiple passages in culture, suggestive of a possible progenitor population and leading us to characterize their lineage potential

Authorship note: Harold A. Chapman, Xiaopeng Li, and Jonathan P. Alexander contributed equally to this work.

Conflict of interest: The authors have declared that no conflict of interest exists. Citation for this article: J Clin Invest. 2011;121(7):2855-2862. doi:10.1172/JCI57673. both ex vivo and in vivo. While this work was in progress, a separate report indicated that epithelial cells isolated from single-cell preparations of whole lungs on the basis of $\alpha 6 \beta 4$ expression have stem-like properties ex vivo, but the location of these cells and their in vivo potential were not defined (8). We here confirmed the existence of a dynamic population of distal epithelial cells and demonstrated, using a lung organoid assay developed in our laboratory that we believe to be novel, the regenerative potential of these cells in vivo.

The replenishment of damaged epithelial cells in the lung parenchyma after injury is thought to depend on proliferation and differentiation of $\mathrm{SPC}^{+}$type II cells. Indeed, the timing and extent of type II cell hyperplasia covering damaged alveolar basement membranes is thought to be a protective process that minimizes the fibrogenic program in the lung $(9,10)$. To address the question of whether type II cells are in fact the major cell type repopulating damaged lung, we developed an in vivo fate-mapping system using tamoxifen-inducible cre recombinase placed within the endogenous SPC locus. These experiments instead revealed a clear role for progenitor cells in lung repair, consistent with our finding that immature epithelial progenitors existed and responded dynamically to injury. Collectively, these findings provide insights into the pathophysiology of lung repair.

\section{Results}

Generation of mice with epithelial-specific loss of $\alpha 6 \beta 4$ integrin. Mice with selective lung epithelial loss of $\alpha 6 \beta 4$ (referred to herein as F $34 S C$ mice) were generated by crossing floxed integrin $\beta 4$ mice with mice carrying the human SPC promoter-rtTA and tetO-CMVCre transgenes (refs. 11, 12, and Supplemental Figure 1A; supplemental material available online with this article; doi:10.1172/ 

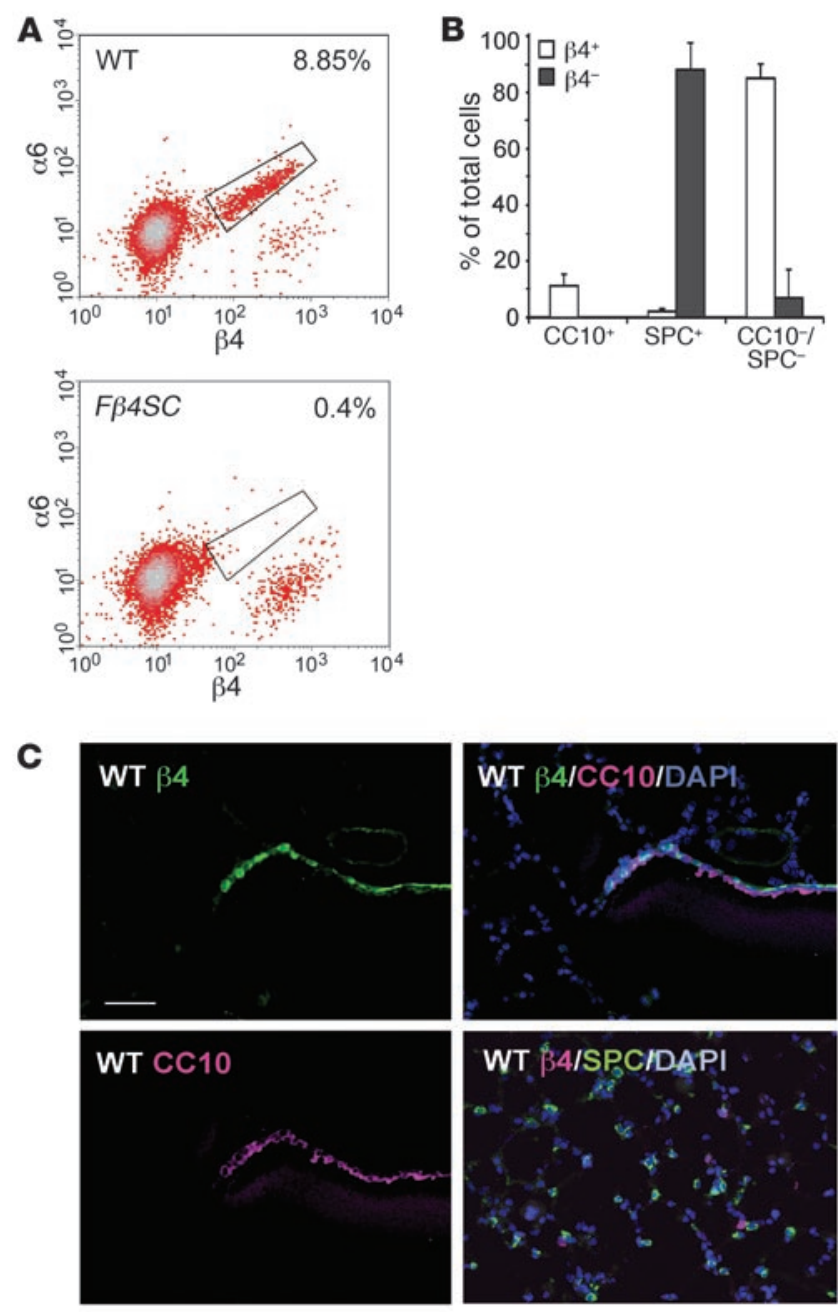

JCI57673DS1). Lung epithelial-specific recombination of triple transgenics was verified by immunostaining for $\beta 4$ (Supplemental Figure 1C). In sections, $\beta 4$ was detected in near-linear basilar staining of airway cells throughout the conducting airways that colocalized with basement membrane laminin 5 (Supplemental Figure 1, C and D). In F $\beta 4 S C$ mice, there was marked loss (range, $75 \%-90 \%, n>12$ ) of integrin staining in all distal conducting airways lined by a layer of cuboidal epithelial cells, but much less or no loss in more proximal airways lined by ciliated cells (Supplemental Figure 1, B-D), consistent with prior reports of cre expression patterns in this transgenic system (13). Inspection of F $\beta 4 S C$ mice indicated that adult mice had no discernible morphological abnormalities in their lungs.

To assess the role of $\alpha 6 \beta 4$ during injury and repair, F $\beta 4 S C$ mice were injected with saline or bleomycin $(2.5 \mathrm{U} / \mathrm{kg})$ intratracheally (12). Lung permeability at baseline and after 5 days was not different between WT and F $\beta 4 S C$ mice (Supplemental Figure 2A). However, beginning at approximately day $6, F \beta 4 S C$ mice began to die, and most succumbed by day 14 , whereas WT mice mostly survived (Supplemental Figure 2B). Inspection of the lungs of F $\beta 4 S C$ mice at 6-10 days after bleomycin injection showed marked sloughing of intact epithelial sheets from the distal airways (Supplemental Figure $2 \mathrm{C}$ ). These findings are consistent with prior observations of integrin $\beta 4$ deficiency in skin and nasal mucosa (7) but indicate

\section{Figure 1}

Identification and location of $\beta 4^{+}$AECs in murine lungs. (A) FACS plot of $\alpha 6$ and $\beta 4$ on AECs from WT and $F \beta 4 S C$ mice. Residual $\beta 4$ staining in the $F \beta 4 S C$ mice is nonspecific secondary antibody. Percentages denote the fraction of cells within the boxed regions. (B) Differential cell counts of $\beta 4^{+}$and $\beta 4^{-}$cells purified by flow cytometry from normal lungs (mean $\pm \mathrm{SD}, n=6$ ). (C) $\beta 4$ immunostaining in WT lungs showing diffusely stained cells near the bronchoalveolar junction along with CC10 staining and the merged images. Note that not all $\mathrm{CC} 10^{+}$cells were $\beta 4^{+}$. Basilar staining of airway $\beta 4$ was apparent near the airway end of the junctional region. There was a lack of costaining of SPC with $\beta 4^{+}$cells in the alveolar region (see also Supplemental Figure 3). Scale bar: $50 \mu \mathrm{m}$.

that, in the lung, epithelial cells appear to remain adhesive until stressed. Although we cannot be sure that the mice die of epithelial sheet sloughing in conjunction with the alveolar injury induced by bleomycin, this complication appears likely to exacerbate the degree of injury and precipitate death.

$F \beta 4 S C$ mice were also examined after airway injury with naphthalene (14). Both WT and F $\beta 4 S C$ mice survived the injury and by day 14-21 showed near-complete recovery of CC $10^{+}$lining cells. Immunostaining confirmed that repopulating Clara cells in the bronchioles of F $\beta 4 S C$ mice had undetectable integrin $\beta 4$. Thus, although $\alpha 6 \beta 4$ was required for strong adhesion, the integrin per se was not required for proliferation and/or differentiation of these cells during repair, consistent with prior studies of $\alpha 6 \beta 4$ deficiency in keratinocytes (11).

Localization of $\alpha 6 \beta 4^{+}$epithelial cells in the lung parenchyma. Flow cytometric analysis of preparations of type II alveolar cells revealed expression of $\alpha 6 \beta 4$ on a small fraction ( $8 \%-10 \%)$ of AECs (Figure $1 \mathrm{~A})$. The presence of $\alpha 6 \beta 4$ in a minor population of AECs raised the possibility that $\alpha 6 \beta 4$ expression might reveal population heterogeneity among AECs, as suggested by prior studies (14-16). $\beta 4^{+}$cells were located both in the bronchoalveolar junction regions and within alveoli (Figure 1C and Supplemental Figure 3). Notably, the pattern of $\beta 4$ staining changed near the junction region and within alveoli. Whereas in the bronchiolar and larger airways, $\beta 4$ staining was quite basilar in hemidesmosomes, in the junctional regions, $\beta 4$ was more diffuse and difficult to detect. Costaining with the Clara cell marker CC10 indicated that only some junctional cells were $\beta 4^{+}$, and these appeared to have lower CC10 expression (Figure 1C). CC10 $-\beta 4^{+}$cells were also found sporadically (less than 1 per $\times 20$ field) in alveolar walls (Figure $1 \mathrm{C}$ and Supplemental Figure 3), where they did not colocalize with pro-SPC. Both junctional and alveolar $\beta 4$ staining was largely lost in F $34 S C$ mice (Supplemental Figure 1C).

To assess the relative airway and alveolar contributions to the $\beta 4^{+}$AEC population, cytospins of freshly isolated cells were analyzed with antibodies to mature epithelial cell types. Cytospins of $\beta 4^{-}$AECs purified by flow cytometry were almost uniformly SPChi and $\mathrm{CC}_{10}$, indicating mature type II AECs, whereas more than $80 \%(83 \% \pm 10 \%, n=4)$ of $\beta 4^{+}$AECs were dim or negative for SPC and CC10 (Figure 1B). These cells were also not stained with the type I AEC marker T1 $\alpha$, and ciliated cells were not observed. A portion of $\beta 4^{+}$cells were strongly $\mathrm{CC} 10^{+}(9 \% \pm 3 \%)$, likely reflecting contamination with Clara cells, which suggests that the $\beta 4^{+}$cell population is itself heterogeneous. Less than $1 \%$ of the purified $\beta 4^{+}$cells were $\mathrm{SPC}^{+}$. Given that the fraction of $\mathrm{CC} 10^{+}$cells within the $\beta 4^{+}$cell population is less than $10 \%$, and yet $\beta 4^{+}$cells repre- 

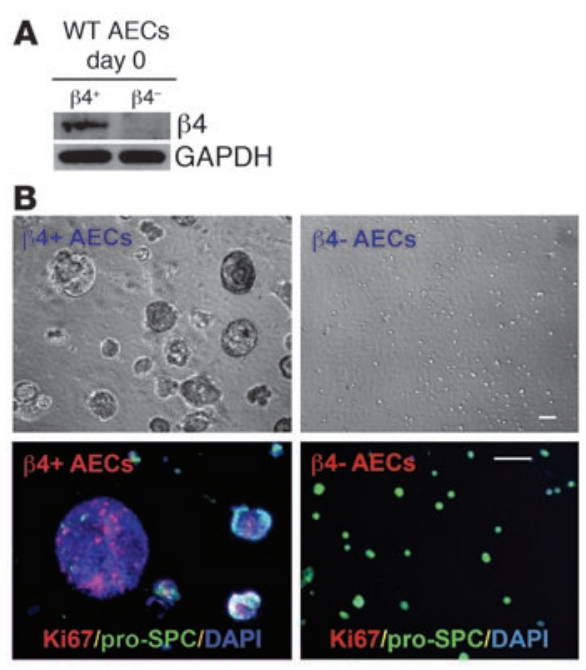

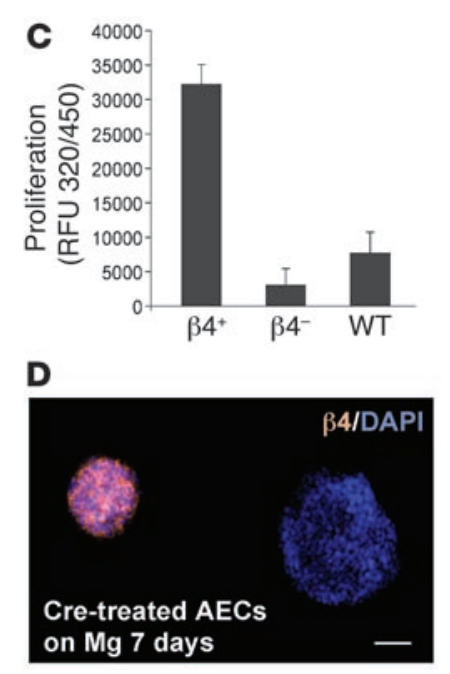

Figure 2

Ex vivo proliferation and clonal expansion of $\beta 4^{+}$AECs.

(A) Immunoblot verifying separation of $\beta 4^{+}$and $\beta 4^{-}$cells.

(B) Phase-contrast images of $\beta 4^{+}$and $\beta 4^{-}$AECs from

WT mice cultured 7 days on Matrigel revealed large clusters only in the $\beta 4^{+}$population. Ki67 and pro-SPC staining of cultured $\beta 4^{+}$and $\beta 4^{-}$AECs revealed proliferation of $\beta 4^{+}$cells and appearance of SPC ${ }^{+}$cells, especially in the smaller clusters. Cells were initially SPClo or SPC-. $\beta 4^{-}$AECs were strongly SPC ${ }^{+}$and had little or no proliferation. The same number of $\beta 4^{+}$and $\beta 4^{-}$cells were initially seeded. (C) BrdU assay at 24 hours (Chemicon) confirmed that greater than $90 \%$ of the proliferation among AECs resided in the $\beta 4^{+}$fraction. WT, cell mixture before sorting. (D) AECs from floxed $\beta 4$ mice treated ex vivo with adenovirus encoding cre recombinase. Developing clusters (>15 per culture well) were either all $\beta 4^{+}$or all $\beta 4^{-}$. Scale bars: $50 \mu \mathrm{m}$. sent $8 \%-10 \%$ of all AECs, we interpret this result to mean that the majority of $\beta 4^{+}$AECs low or negative for SPC and CC10 in our preparations must come from the alveolar compartment. To date, we have no method to reliably separate $\beta 4^{+}$cells located in terminal airways/bronchoalveolar junctions from those within alveoli; thus, the term $\beta 4^{+} A E C$ used herein encompasses mainly alveolar cells, but also terminal airway/junctional cells.

$\beta 4^{+}$AECs proliferate and expand clonally ex vivo. $\beta 4^{+}$cells were purified from standard AEC preparations by positive E-cadherin antibody selection followed by sorting for $\beta 4^{+}$cells. Immunoblotting confirmed that the $\beta 4^{+}$and $\beta 4^{-}$cell populations were distinct (Figure $2 \mathrm{~A}) . \beta 4^{+} \mathrm{AECs}$ cultured on $\mathrm{Mg}$ developed large cell colonies within 7 days, whereas $\beta 4^{-}$AECs remained as single cells or small clusters (Figure 2B). Ki67 staining confirmed proliferation within the developing colonies of $\beta 4^{+}$AECs (Figure $2 \mathrm{~B}$ ), and quantitative BrdU incorporation revealed that greater than $90 \%$ of the proliferative potential of AECs resided in the $\beta 4^{+}$fraction (Figure $2 \mathrm{C}$ ). The colonies of $\beta 4^{+}$cells could be recovered (by pipetting) and passaged onto new $\mathrm{Mg}$ for at least 6 passages. Differential cell counts of dispersed, single colonies $(n=12)$ that had expanded over 7 days revealed colony heterogeneity. The colonies were greater than $60 \%$ $\mathrm{SPC}^{-} \mathrm{CC} 10^{-}$, but almost all colonies now also contained both $\mathrm{SPC}^{+}$ $(22 \% \pm 30 \%)$ and $\mathrm{CC} 10^{+}(13 \% \pm 6 \%)$ cells. Accounting for the large $\mathrm{SD}$ for this marker, 2 colonies were exclusively $\mathrm{SPC}^{-} \mathrm{CC} 10^{-}$, and 2 colonies were exclusively $\mathrm{SPC}^{+}$. A small percentage $(3 \% \pm 3 \%)$ was $\mathrm{SPC}^{+} \mathrm{CC} 10^{+}$, a cell type never observed among the freshly isolated $\beta 4^{+}$AECs. Notably, approximately $10 \%-15 \%$ of the colony cells were $\beta 4^{-}$, indicative of some tendency to lose this marker in culture. Colony formation by $\beta 4^{+}$AECs plated on irradiated embryonic feeder fibroblasts for 1-2 weeks was not observed.

To determine the role of integrin $\beta 4$ in the proliferation of $\beta 4^{+}$ AECs, $\beta 4^{+}$AECs were isolated from mice homozygous for the nonrecombined conditional $\beta 4$ allele and treated with cre recombinase for 72 hours ex vivo. Remarkably, colonies that emerged in 7 days were exclusively $\beta 4^{-}$or $\beta 4^{+}$, and the sizes of the colonies were indistinguishable (Figure 2D), which indicates that these colonies likely developed independently of $\beta 4$ and from single cells. To further explore the latter point, $\beta 4^{+} \mathrm{AECs}$ were purified from transgenic mice constitutively expressing either GFP or tomato red fluorescent protein. When single $\beta 4^{+}$cell suspensions from either mouse were mixed and placed in culture, all of the individual colonies $(n=65)$ were exclusively red or green, indicative of clonal expansion rather than cell aggregation (Supplemental Figure 4). This observation, together with the finding of both $\mathrm{CC} 0^{+}$and $\mathrm{SPC}^{+}$cells in $1 \beta 4^{+}$colony, implies that single SPC ${ }^{-}$or $\mathrm{CC}^{-} 0^{-} \beta 4^{+} \mathrm{AECs}$ can differentiate in culture into cells with markers of either airway or alveolar cells. We next attempted to test the progenitor potential of $\beta 4^{+} \mathrm{AECs}$ in vivo.

Development of an in vivo organoid assay of lung development. Prior studies have demonstrated that dissociated mouse E14.5 lung cells cultured as high-density mixed cell aggregates (lung organoids) at an air-fluid interface can form lung histotypic structures, including alveolar-like spheres, lined by appropriately differentiated epithelial cells (17). We have improved upon this method to achieve extensive development of lung organoids in vivo that recapitulated normal development. We implanted high-density mixed cell aggregates (organoids) prepared from single-cell suspensions from E14.5 lungs underneath the renal capsule of immunodeficient (nude) mice. These grafted lung organoids grew and became easily visible after 6 days (Figure 3, A and B). This model was highly reproducible, and almost all engraftments resulted in fully developed organoids. Notably, a vasculature developed within the structure and connected with the host vasculature, as rbcs were apparent in tubular structures within the grafts (Figure 3C). Graft development required both epithelial and mesenchymal cells, which indicates that specific cell populations can be examined for their developmental potential in this assay.

Examination of histological sections of the grafts at days 2-6 showed that the grafts underwent a series of developmental stages resembling that of normal embryonic lung (Figure 3D): day 2 grafts resembled pseudoglandular-stage lungs, containing epithelial tubes surrounded by abundant mesenchyme; day 4 grafts resembled canalicular-stage lungs, containing epithelial tubes lined by columnar epithelial cells typical of proximal airways as well as branching structures lined by more flattened epithelial cells typical of distal airspaces; and day 6 grafts contained structures resembling saccules composed of interconnected airspaces lined by flattened epithelial cells, typical of E18.5 lungs. To directly test whether the embryonic singlecell suspensions are both organizing and differentiating under the kidney capsule, organoid sections were immunostained for CC10, SPC, and Pecam-1. E14.5 lungs showed SPC in airway epithelial cells (Supplemental Figure 5B), whereas in the day 5 organoid, SPC was found in cells lining saccular structures (Figure 3, E and F, and 
A

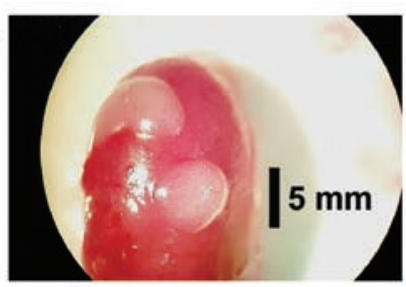

B
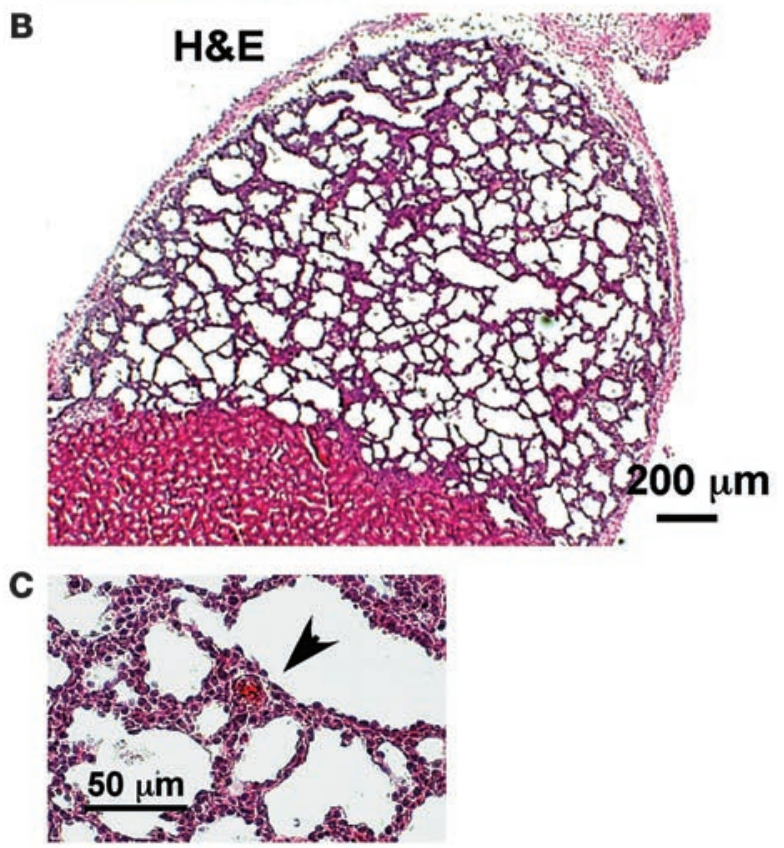

D

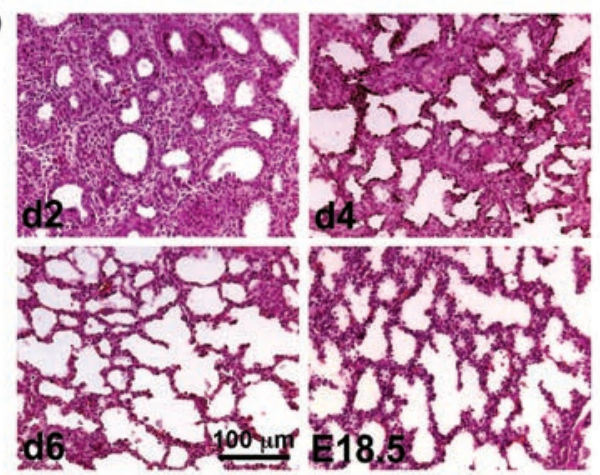

E

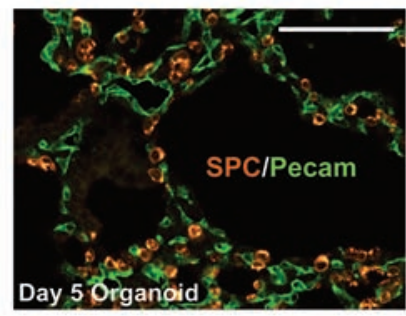

$\mathbf{F}$

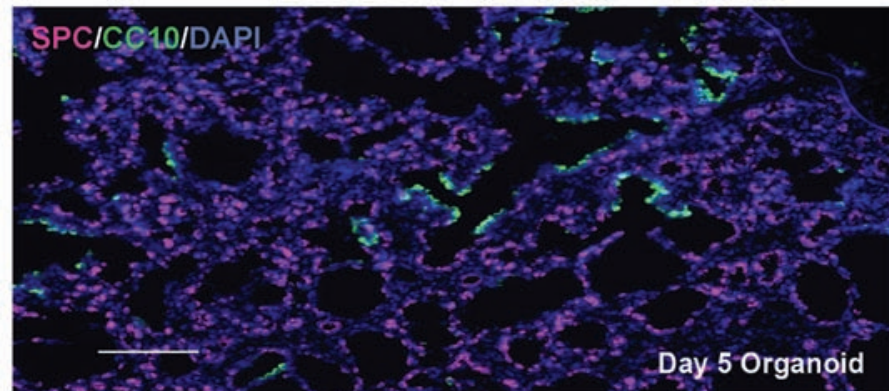

Figure 3

Embryonic lung organoid assay. (A) Aggregates of single-cell preparations of E14.5 lung cells placed under renal capsules developed visible structures (white) after 5 days in vivo. The red structure is the underlying kidney. (B) Low-power view of H\&E stains of the organoid revealed fluid-filled lacy structure under the capsule with visible airway-like and saccular elements. (C) Higher-power view revealed rbcs in blood vessel (arrowhead), suggestive of vascular continuity with the host kidney. (D) H\&E-stained sections of day 2, day 4, and day 6 organoids showing development of the organoid to the saccular stage by day 6, similar to that of the E18.5 lung (see Supplemental Figure 5). (E) Distribution of SPC and Pecam-1 in a day 5 organoid. See Supplemental Figure 5 for distribution of Pecam-1 and SPC and absence of CC10 staining in E14.5 lungs. (F) CC10 and pro-SPC double staining of day 5 organoid sections. Airway-like regions led to SPC+ saccules with few, if any, SPC ${ }^{+} \mathrm{CC}^{+} 0^{+}$ cells. Scale bars: $5 \mathrm{~mm}$ (A); $200 \mu \mathrm{m}$ (B); $50 \mu \mathrm{m}$ (C and E); $100 \mu \mathrm{m}$ (D and F).

Figure 4, A and C), consistent with the pattern of SPC expression in saccular-stage lungs. CC10 was not detectable in day 14.5 lungs (Supplemental Figure 5B), since it is normally found only at low levels starting at E15.5 and increases thereafter $(18,19)$. In day 5 organoids, vivid CC10 staining was apparent in cuboidal epithelial cells (Figure 3F and Figure 4B), consistent with expression in bronchiolar epithelial cells. Strikingly, there were virtually no $\mathrm{SPC}^{+} \mathrm{CC} 10^{+}$ cells and even little mixing of single-positive cells. Regions of the developing organoid were commonly $\mathrm{CC} 10^{+}$airway-like, leading into "blind" $\mathrm{SPC}^{+}$saccules (Figure $3 \mathrm{~F}$ ). True quantification of the fraction of airway and saccular structures was not attempted, but $\mathrm{SPC}^{+}$saccules were always more abundant than $\mathrm{CC} 10^{+}$airway-like structures. Whether all saccules were connected to an airway-like structure could not be reliably assessed. We next tested the potential of adult $\beta 4^{+}$or $\beta 4^{-}$AECs to incorporate into and differentiate in the developmental environment of a lung organoid.

$\beta 4^{+}$adult AECs differentiate in vivo. Organoids were made from E14.5 lung cells depleted of epithelial (E-cadherin ${ }^{+}$) cells and reconstituted with purified $\beta 4^{+}$or $\beta 4^{-}$AECs admixed with E-cadherin ${ }^{+}$embryonic cells in various ratios and grafted under the renal capsule for 5-6 days. Preliminary experiments indicated that a 1:10 ratio of adult AECs to embryonic epithelial cells resulted in organoids similar in size to unperturbed E14.5 cell suspensions. Under these conditions, $\mathrm{GFP}^{+} \beta 4^{-} \mathrm{AEC}$ only occasionally persisted as single cells in the organoids, but were unable to form discernible structures (Supplemental Figure $5 \mathrm{~A}$ ). However, $\mathrm{GFP}^{+} \beta 4^{+} \mathrm{AECs}$ were found to organize and incorporate extensively into the developing lung organoids (Figure 4A). As evident in the scanning view (Figure 4A), $\mathrm{GFP}^{+}$cells were not distributed as single cells, but instead appeared as foci or clusters of cells (Figure 4, B and C). Closer inspection revealed that $\mathrm{GFP}^{+} \beta 4^{+}$AECs developed into either discrete $\mathrm{SPC}^{+}$saccules or $\mathrm{CC} 10^{+}$airway-like structures. There were only rare $\mathrm{SPC}^{+} \mathrm{CC} 10^{+}$cells and few SPC-CC10 ${ }^{-}$cells: most $\mathrm{GFP}^{+} \beta 4^{+} \mathrm{AEC}$ were at least weakly $\mathrm{SPC}^{+}$, if not $\mathrm{CC} 10^{+}$. Intriguingly, the organoid structures developing from mixtures of embryonic/adult $\mathrm{GFP}^{+} \beta 4^{+}$cells (10:1) were almost exclusively either wholly adult cell derived or wholly embry- 
A

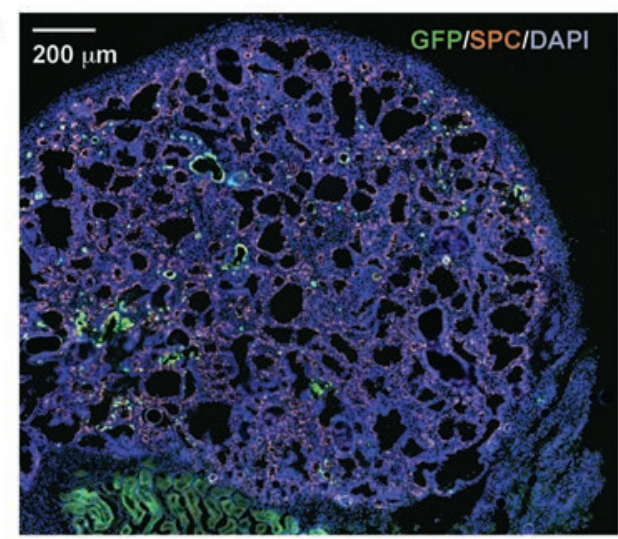

B

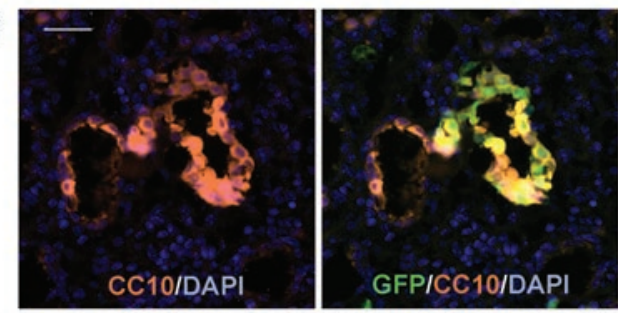

C

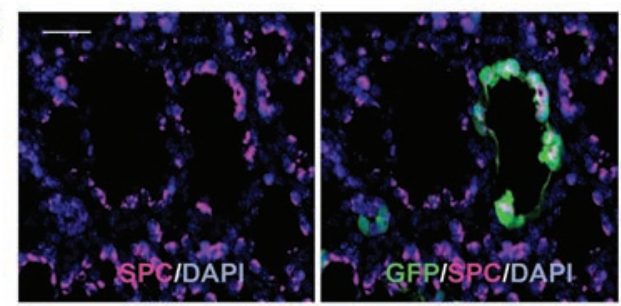

onic, indicative of a clear capacity for self-organization. This point is illustrated in Figure 4B, which juxtaposes 2 airway-like structures lined by $\mathrm{CC} 10^{+}$cells, one fully of embryonic origin and the other fully derived from $\mathrm{GFP}^{+}$cells. A similar pattern was observed with $\mathrm{SPC}^{+}$saccules (Figure 4C). Collectively, these data indicate that adult $\beta 4^{+}$AECs can both differentiate ex vivo and regenerate epithelial structures in vivo. The almost exclusive differentiation into distinct $\mathrm{SPC}^{+}$or $\mathrm{CC} 10^{+}$structures, even though $\beta 4^{+}$colonies ex vivo were multipotential (Figure 2), indicates a strong influence of the organoid microenvironment on differentiation in vivo, a conclusion consistent with the spatial separation of CC10 and SPC expression in the day 6 embryonic organoid itself (Figure 3D).

$\beta 4^{+} A E C$ s expand and populate alveolar regions after lung injury. We next asked whether the pool size of $\beta 4^{+} \mathrm{AECs}$ changes in response to injury. Mice were exposed to bleomycin $(2.5 \mathrm{U} / \mathrm{kg})$, and AECs were isolated after 14 days. Flow cytometry consistently showed an increased fraction of $\beta 4^{+}$AECs $7-10$ days after bleomycin injury (Figure 5B). In 5 experiments, the recovered $\beta 4^{+}$cells increased on average from approximately $10 \%$ of the total AECs in saline-treated mice to $27 \% \pm 10 \%$ in bleomycin-injured mice. This was reflected in increased amounts of integrin $\beta 4$ assessed by immunoblotting of AECs recovered from bleomycin- compared with saline-injected mice (Figure 5A). Cytospins of the cells recovered from injured mice following sorting for $\beta 4$ revealed substantially more $\mathrm{CC}^{+} 0^{+}$ cells $(20 \%)$, likely reflecting either increased airway contamination or bronchiolization of the alveoli, which was evident in sections of injured lung (Figure 5, D and E). Still, the majority of recov-

\section{Figure 4}

Development of adult $\beta 4^{+}$AEC-derived structures in lung organoids. (A) Low-power view of organoid with scattered clusters of GFP+ $\beta 4^{+}$ AECs. Multiple images (original magnification, $\times 20$ ) were captured and tiled into a single composite image. (B) Adjacent airway-like $\mathrm{CC} 10^{+}$ structures, one completely GFP-, the other entirely GFP+. Such dichotomy in the origin of $\mathrm{CC}_{10} 0^{+}$structures was the most common pattern in these mixed organoids. (C) SPC+ saccules, again showing entire saccular structures either GFP+ or GFP- $^{-}$. Although this was the most common pattern, occasional saccules did contain both embryonic and adult SPC+ cells. Scale bars: $200 \mu \mathrm{m}(\mathbf{A}) ; 20 \mu \mathrm{m}$ (B and C).

ered $\beta 4^{+}$AECs after bleomycin injury remained low or negative for CC10 and SPC. Moreover, unlike the case with normal lungs, $\mathrm{SPC}^{+} \beta 4^{+}$cells ( $4 \%$ ) were recovered from bleomycin-injured lungs. Consistent with this observation, some alveolar $\beta 4^{+}$cells in injured lungs also stained for SPC (Figure 5C).

Is there evidence of AEC Progenitor cell differentiation after lung injury? Given the increased numbers of $\beta 4^{+} \mathrm{AECs}$ low or negative for CC10 and SPC - likely with progenitor potential - after parenchymal injury (Figure 5), we asked whether maintenance of the type II cell population after major injury involves AEC progenitors. To address this issue, we created an inducible system for fate-mapping alveolar type II cells using homologous recombination. The terminal stop codon of the endogenous mouse SPC gene (exon 5; Figure 6A) was replaced with an estrogen-dependent cre cDNA (CreERT2) preceded in frame by a E2A peptide sequence to interrupt translation between SPC and cre mRNAs coexpressed on a single transcript (20). A Nde1 site was introduced to facilitate ES cell screening, and correctly targeted clones were generated, as confirmed by Southern blotting (Figure 6B). ES cell-derived mice homozygous for the mutation showed normal SPC levels by immunoblotting, and cross-breeding of these mice with a cre-dependent transmembrane GFP (loxp-tm-TR/GFP) reporter strain revealed tamoxifeninducible GFP expression specifically in type II cells (Figure 6C). In the absence of tamoxifen, less than $1 \mathrm{GFP}^{+}$cell per $\times 20$ field was detected, whereas $50 \%-60 \%$ of all type II AECs were $\mathrm{GFP}^{+} 7$ days after 2 total 4-hydroxy-tamoxifen i.p. injections $(0.5 \mathrm{mg}) 24$ hours apart (Figure 6C). No GFP expression was observed in airway cells of tamoxifen-exposed mice, as expected.

A cohort of adult littermates expressing SPCcre-ER and loxp-tmTR/GFP was injected with tamoxifen, and lungs were examined 7 or 30 days later. In some mice, bleomycin was injected intratracheally 14 days prior to sacrifice. The fraction of $\mathrm{GFP}^{+} \mathrm{SPC}^{+}$cells was quantified by independent observers examining at least 10 $\times 20$ fields of at least 3 sections of lung. The fraction of $\mathrm{GFP}^{+} \mathrm{SPC}^{+}$ cells 30 days after tamoxifen exposure $(60 \% \pm 10 \%)$ was not discernibly different from that observed at 7 days (Figure 6D). However, the fraction of $\mathrm{GFP}^{+} \mathrm{SPC}^{+}$cells in fibrotic areas after bleomycin was markedly reduced to less than $15 \%$ (Figure 6, D and E). This was not caused by global lack of cre expression, because some less-injured lung areas of the same mouse had vivid GFP expression in $\mathrm{SPC}^{+}$cells (Figure 6E). Hyperplastic type II AECs lining alveoli within injured areas were consistently GFP- (Figure 6E and Supplemental Figure 6). The reporter strain used in this analysis, loxp-tm-TR/GFP, also expressed tomato red fluorescent protein from the same allele in nonrecombined cells, and red fluorescence was easily detected throughout injured areas (Figure 6F), which indicated that the reporter gene itself was not silenced in bleomycin-injured lungs. Therefore, the data imply that $\mathrm{SPC}^{+}$type II 

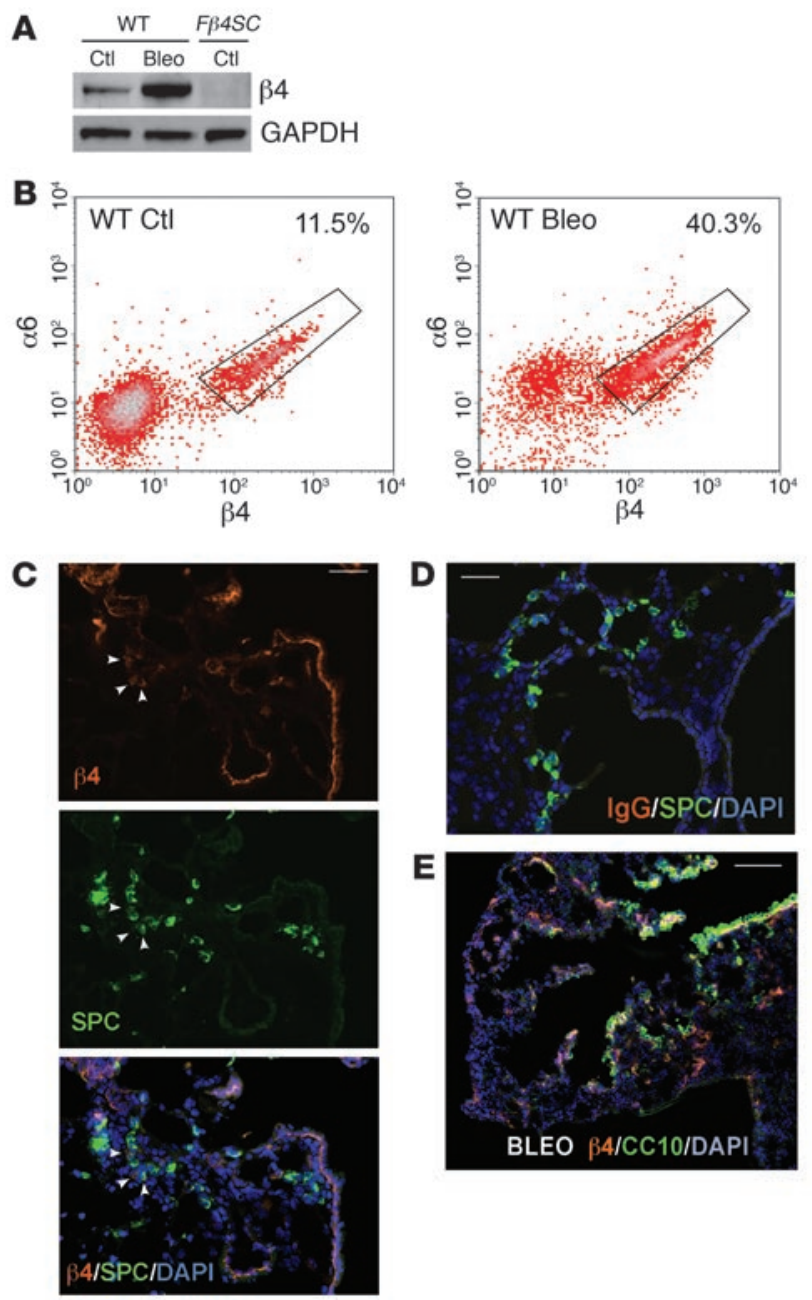

cells in day 14 bleomycin-damaged areas are largely derived from non-SPC-expressing progenitor cells existing at the time of injury. Similar results were observed in 4 independent experiments.

\section{Discussion}

There is substantial evidence that the adult distal lung parenchyma, composed of bronchioles and alveoli, contains relatively quiescent epithelial cells that have proliferative and progenitor capacity (15, 21,22 ). The current thinking is that Clara cells (or variant Clara cells with low CC10 expression) and alveolar type II cells are the main cell types with progenitor function in the distal lung parenchyma $(22,23)$. Our findings here showed that in the normal lung, $\alpha 6 \beta 4$ expression marked a strikingly fixed population of non-SPCexpressing AECs that possessed the capacity for proliferation and further differentiation ex vivo and regeneration of lung structures in vivo. These $\beta 4^{+}$AECs were distinguished from Clara cells or alveolar type II cells by their lack of high levels of differentiation markers and the lack of proliferation of mature cells in culture (Figure 2). $\beta 4^{+}$AECs were found to reside in the bronchoalveolar junction regions, but also sporadically in alveolar walls. Based on the small fraction of $\mathrm{CC} 10^{+}$cells within the isolated $\beta 4^{+} \mathrm{AEC}$ population and the observation that $\beta 4^{+}$AECs accounted for almost $10 \%$ of total AECs recovered from normal lungs, the bulk of these cells appeared to be in the alveolar compartment, where they may be difficult to

\section{Figure 5}

Expansion of $\beta 4^{+}$AECs in lungs of bleomycin-treated mice. (A) Immunoblot for $\beta 4$ in purified $\beta 4^{+}$AECs from mice injected with saline (Ctl) or bleomycin (Bleo) 10 days earlier. (B) The higher proportion of $\beta 4^{+}$AECs from bleomycin-injected mice was confirmed by flow cytometry of $\beta 4$ and $\alpha 6$. Percent cells within boxed regions is indicated. (C) $\beta 4$ and SPC staining of a lung section from mouse injected intratracheally with bleomycin 14 days earlier. Arrowheads denote SPC $+\beta 4^{+}$cells. (D) IgG-negative control for $\beta 4$ immunostaining on a lung section adjacent to that in C. (E) Compared with normal lungs, there were many more $\beta 4^{+}$cells in the alveolar compartment 21 days after bleomycin, but there were also $\mathrm{CC} 10^{+} \beta 4^{+}$cells extending into the alveolar region from the distal airway/bronchoalveolar junction. Scale bars: $50 \mathrm{~mm}$ (C and D); $100 \mu \mathrm{m}$ (E).

detect in situ because of diffuse distribution of $\alpha 6 \beta 4$. Diffuse staining of $\beta 4$, indicative of dissolution of hemidesmosomes, has been previously observed in tumor cells (24). Although this population was stable in normal lungs, its proportion increased in bleomycininjured murine lungs. In vivo, there was expansion of $\alpha 6 \beta 4^{+}$epithelial cells, some of which were either $\mathrm{CC} 10^{+}$or $\mathrm{SPC}^{+}$, into or within alveolar spaces during the fibrogenic phase of bleomycin injury. Finally, we provided the first direct evidence to our knowledge that maintenance of type II AECs after major lung injury prominently involved AEC progenitor differentiation and not simply expansion of preexisting type II cells. Although we cannot prove at this point that $\beta 4^{+}$AECs within alveoli are the only progenitor source of type II AECs within the injured lung parenchyma, our data identify a strong candidate stem/progenitor cell that could account for these data. Further definition of the requirement for AEC progenitors in lung repair after different types and degrees of lung injury, and the specific requirement for $\beta 4^{+}$AECs in this process, are important areas for further study.

While the present work was in progress, McQualter and colleagues reported the isolation of stem-like cells from adult mouse lungs using integrin $\alpha 6 \beta 4$ expression as a defining biomarker (8). Our findings support and extend this work. In the McQualter report, the starting material was a single-cell suspension of whole adult lung, but the location of the $\alpha 6 \beta 4^{+}$cells with stem-like properties in the lung could not be defined. No immunostaining for $\beta 4$ in the bronchoalveolar regions or alveoli was observed. In contrast, the starting material for the present study were cells from the distal airways and alveoli. We were able to capitalize on the generation of a lung epithelial-specific $\beta 4$-null mouse to verify that immunostaining for $\beta 4$ in the bronchoalveolar and alveolar compartments was real. Nonetheless, in situ immunostaining appeared insensitive, and it is likely that at least a portion of the cells isolated by McQualter and colleagues is identical to the $\beta 4^{+}$AECs described in this study.

This line of investigation emanated from the finding that only a fraction of primary AECs cultured ex vivo on fibronectin underwent epithelial-to-mesenchymal transition (EMT) when exposed to active TGF- $\beta 1$, and this population proved to be mainly $\beta 4^{+}$AECs (Supplemental Figure 7). There is accumulating evidence of functional similarities between the capacity of cells to undergo EMT and their display of stem-like properties (25). Our observations raise the possibility that EMT previously reported in vivo in mouse models of fibrosis (26) - and commonly thought to result from type II cells undergoing EMT - could mainly result from the enhanced plasticity of the $\beta 4^{+}$AEC progenitors located in the normal lung and expanded after bleomycin injury in response to TGF- $\beta 1$. 
A

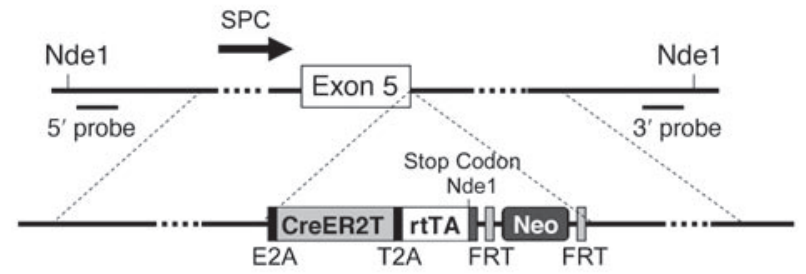

B

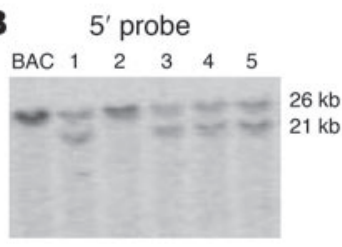

C

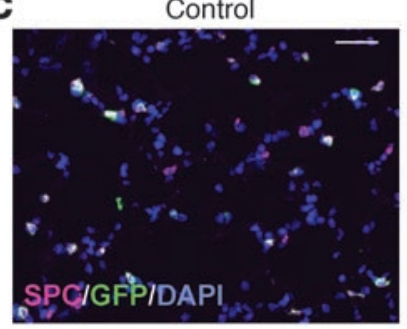

D

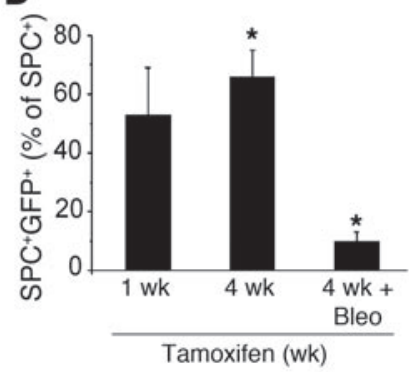

E

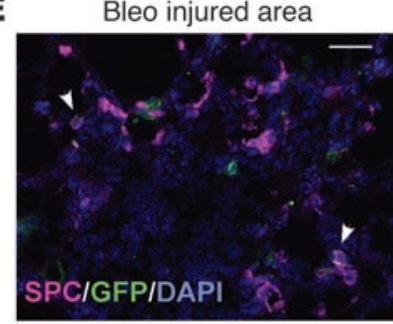

Bleo uninjured area

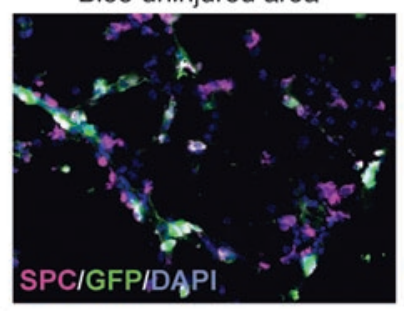

$\mathbf{F}$

2 weeks after Bleo

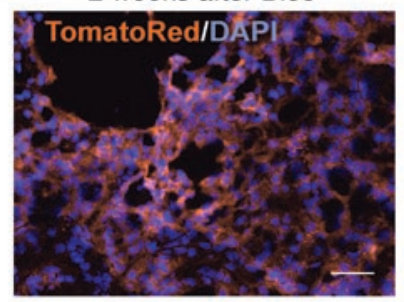

The in vivo organoid assay we developed and used herein is, to our knowledge, the first in vivo transplantation assay beginning with single-cell suspensions that recapitulates lung morphogenesis. Among the many possible applications of this assay, of particular interest here is our finding that adult $\beta 4^{+}$AECs selforganized into structures bearing a single lineage marker in the day 6 organoids (Figure 4). How these cells sort themselves from the more abundant embryonic epithelial cells and self-organize as they differentiate is currently unknown. The adult $\beta 4^{+}$AECs used included cells located at the bronchoalveolar junction and within alveoli. One limitation of these experiments is that we cannot separate $\beta 4^{+}$AECs present in the bronchoalveolar junction regions from those within alveoli, and thus we cannot determine to what degree cells from either site contribute to the $\mathrm{CC}^{+} 0^{+}$and $\mathrm{SPC}^{+}$structures that developed in vivo (Figure 4). Hence, we could not formally exclude the possibility that bronchoalveolar $\beta 4^{+}$cells, rather than $\beta 4^{+}$AECs, account for all progenitor activity in the organoid assay. However, we believe the findings that almost all colonies derived from single $\beta 4^{+}$cells ex vivo contained $\mathrm{SPC}^{+}$cells (Figure 2 ) and that most $\beta 4^{+}$cells appeared to reside within alveoli, where progenitor activity for type II cell maintenance appears important (Figure 6), are more consistent with the view that $\beta 4^{+}$ AECs in both locations have progenitor potential, but their dif-

\section{Figure 6}

Role for SPC- progenitor cells in type II cell replenishment after bleomycin-induced injury. (A) Insertion site of CreER2T, rtTA, and Neo cassette cDNAs into the stop codon of the endogenous SPC gene. A new stop codon was created at the $3^{\prime}$ end of rtTA, which was not used in these experiments. The Neo cassette had not been removed. See Supplemental Methods for details. (B) Southern blot of ES cell DNA using 5' probe positioned as indicated in A revealed 4 correctly targeted clones (21-kb band in the Nde1 digest). (C) Merged image of pro-SPC and endogenous GFP fluorescence of SPCcreT2/loxp-tmTR/GFP lungs 7 days after i.p. injection of $1 \mathrm{mg} 4 \mathrm{OH}$-tamoxifen. (D) Quantification of GFP $+\mathrm{SPC}^{+}$cells as a fraction of total SPC+ cells in noninjured lungs (7 or 30 days after tamoxifen) or in injured regions of day 30 littermates injected with bleomycin 14 days before sacrifice. Greater than 200 SPC $^{+}$cells in more than $10 \times 20$ fields were counted in 3 sections per lung. Fibrotic areas were identified by crowded nuclei and distorted/collapsed alveolar architecture and confirmed by $\alpha$-SMA staining of adjacent sections. ${ }^{*} P<0.0001$. (E) Merged images of proSPC and GFP fluorescence of fibrotic and less-injured or uninjured lung areas of SPCcreT2/loxp-tm-TR/GFP mice 30 days after tamoxifen and 14 days after bleomycin. Arrowheads denote $2 \mathrm{GFP}+{ }^{+} P^{+}{ }^{+}$cells in fibrotic region. (F) Endogenous red fluorescence of bleomycin-injured loxp-tm-TR/GFP lung in the absence of cre. Scale bars: $50 \mu \mathrm{m}$.

ferentiation in vivo is restricted by their niches. This conclusion is consistent with prior in vivo evidence that bronchoalveolar $\mathrm{CC}^{+} 0^{+}$ cells do not give rise to $\mathrm{SPC}^{+}$cells in vivo, even after injury (22). It is likely that cells similar to the $\beta 4^{+}$AECs exist in more than one anatomical niche, and it remains to be defined to what degree more proximal airway basal cells or other bronchiolar epithelial cells with stem cell properties $(27,28)$ function similarly to $\beta 4^{+}$ AECs in the organoid assay.

The finding that a stable but dynamically responsive population of immature progenitor cells exists in the parenchyma of adult lungs and likely contributes to alveolar repair has implications for the pathogenesis of common lung diseases in which tissue remodeling is a dominant feature. The current thinking has been that $\mathrm{SPC}^{+}$ type II cells are the main stem cells of the alveolar compartment $(9,23)$. Our observations suggest instead that, at least in the context of severe insult, preexisting SPC- epithelial progenitors expand and contribute substantially to repair. However, little is known about the degree to which such cells contribute to resistance to alveolar unit loss under stress, such as with cigarette smoking, or promote pathological mesenchymal expansion during fibrogenesis. Understanding the determinants of $\beta 4^{+} \mathrm{AEC}$ population size and how these cells expand, self-organize, and differentiate along particular lineages should provide further insights into the processes of lung repair, the foundation for better therapeutics.

\section{Methods}

Genetically modified mice. $\beta 4$ loxP mutant mice and SPC and Cre transgenic mice were described previously $(11,26)$. Pregnant females were maintained on doxycycline throughout gestation, and resulting litters were genotyped as previously described $(11,26)$. Both $\beta$-actin promoter-driven GFP-expressing (Tg[CAG-EGFP]B5Nagy) and $\beta$-actin-driven tomato red/

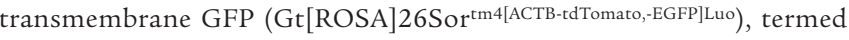
loxp-tm-TR/GFP, transgenic mice were obtained from Jackson Laboratories. Mice were engineered to express tamoxifen-responsive cre-ERT2 and rtTA in frame with SPC but separated by "self-cleaving" E2A/T2A peptide sequences (Figure 6A) using standard recombineering methodologies and 129/C57BL6 hybrid ES cells (iGeniousTL Inc.). See Supplemental Methods 
for details. All animal procedures were approved by the Institutional Animal Care and Use Committee of UCSF. All mice were bred and maintained in a specific pathogen-free environment.

Mouse type II and $\alpha 6 \beta 4 \mathrm{AEC}$ isolation. Isolation of primary AECs was performed as previously described (8); see Supplemental Methods for details. In most experiments, suspended AECs from standard AEC preparations were further purified by positive selection for E-cadherin using MACS magnetic bead technology. These cells were then sorted for $\beta 4$ expression with a rat monoclonal $\beta 4$ antibody directly conjugated with Alexa 647 using a MoFlo Cell Sorter (Dako) within the UCSF Flow Cytometry Core Facility.

Immunofluorescence microscopy and flow cytometry. Isolated cells and 5- to $7-\mu \mathrm{m}$ cryosections were stained by immunofluorescence, $\mathrm{X}$-gal, or H\&E as previously described (26). Cells were analyzed by flow cytometry using an LSR II cytometer (BD Biosciences) and FlowJo.

Where indicated in the figure legends, mosaic images were generated from multiple $\times 20$ images captured on a Zeiss Axio upright fluorescent microscope and tiled using $10 \%$ image overlap by Axiovision 4.7 software.

Immunoblot. Tissue and cells were lysed in RIPA buffer $(150 \mathrm{mM} \mathrm{NaCl}$, $50 \mathrm{mM}$ Tris, $\mathrm{pH} 8.0,1 \%$ Triton $\mathrm{X}-100,0.5 \%$ sodium deoxycholate, $0.1 \%$ SDS, supplemented with protease and phosphatase inhibitors) and analyzed by immunoblotting (26).

Preparation of mixed embryonic lung cell organoids. Mouse E14.5 lungs were dissected, diced, and digested in $0.2 \%$ collagenase/dispase at $37^{\circ} \mathrm{C}$ for 45 minutes. The digests were washed and resuspended in medium (BGJB with $5 \% \mathrm{FBS}, 1 \mathrm{mg} / \mathrm{ml}$ ascorbic acid, $1 \times$ penicillin/streptomycin). Debris was removed from mechanically dissociated cells through a $70-\mu$ m nylon cell strainer. The single-cell suspension was then centrifuged, washed, resuspended in culture medium, and counted. For each organoid, $2 \times 10^{6}$ embryonic cells in single-cell suspension were concentrated by centrifugation, and the suspension (approximately 5-8 $\mu \mathrm{l}$ ) was pipetted onto a 13-mm polycarbonate filter with $8-\mu \mathrm{m}$ pore size floating on culture medium. The organoids were placed in a $\mathrm{CO}_{2}$ incubator overnight, and then implanted under the renal capsule, as described previously (29). To assess the potential of adult $\beta 4^{+}$AECs to incorporate into the organoids, $\beta 4^{+}$ AECs were isolated as described above and expanded for 7 days by culture on $\mathrm{Mg}$, after which single-cell suspensions were mixed in varying ratios (1:10-1:100) with purified E-cadherin ${ }^{+}$E14.5 cells and reconstituted with embryonic mesenchymal E14.5 cells (E-cadherin-) prior to engraftment.

Statistics. Variance for all group data is expressed as \pm SD. For evaluation of group differences, the unpaired 2-tailed Student's $t$ test was used assuming equal variance. A $P$ value less than 0.05 was accepted as significant.

\section{Acknowledgments}

This work was supported by the Burroughs Welcome Foundation (to J.P. Alexander) and by NIH grants HL67204 (to H.A. Chapman), HL75680 (to T.H. Vu), and HL73823 (to T.H. Vu). The authors thank Barry Stripp for providing goat anti-CC10 antibodies, Nigel Kileen and Chris Allen (UCSF) for helpful discussions, and Janet Chen and Maria Sereda for technical assistance with the animal colony.

Received for publication February 21, 2011, and accepted in revised form May 4, 2011.

Address correspondence to: Thiennu H. Vu, University of California, San Francisco, Box 2911, San Francisco, California 94143, USA. Phone: 415.514.4266; Fax: 415.514.4365; E-mail: thiennu.vu@ ucsf.edu. Or to: Harold A. Chapman, University of California, San Francisco, 513 Parnassus Ave, HSE 201, San Francisco, California 94143, USA. Phone: 415.514.0896; Fax: 415.502.4995; E-mail: hal.chapman@ucsf.edu.
1. Coraux C, Meneguzzi G, Rousselle P, Puchelle E, Gaillard D. Distribution of laminin 5, integrin receptors, and branching morphogenesis during human fetal lung development. Dev Dyn. 2002; 225(2):176-185.

2. Nguyen NM, Senior RM. Laminin isoforms and lung development: all isoforms are not equal. Dev Biol. 2006;294(2):271-279.

3. O'Reilly AM, Lee HH, Simon MA. Integrins control the positioning and proliferation of follicle stem cells in the Drosophila ovary. J Cell Biol. 2008; 182(4):801-815.

4. Sheppard D. Epithelial integrins. Bioessays. 1996; 18(8):655-660.

5. Wilhelmsen K, Litjens SH, Sonnenberg A. Multiple functions of the integrin alpha6beta 4 in epidermal homeostasis and tumorigenesis. Mol Cell Biol. 2006;26(8):2877-2886.

6. Niessen CM, van der Raaij-Helmer MH, Hulsman $\mathrm{EH}$, van der Neut R, Jonkman MF, Sonnenberg A. Deficiency of the integrin beta 4 subunit in junctional epidermolysis bullosa with pyloric atresia: consequences for hemidesmosome formation and adhesion properties. J Cell Sci. 1996;109(pt 7):1695-1706.

7. van der Neut R, Krimpenfort P, Calafat J, Niessen $\mathrm{CM}$, Sonnenberg A. Epithelial detachment due to absence of hemidesmosomes in integrin beta 4 null mice. Nat Genet. 1996;13(3):366-369.

8. McQualter JL, Yuen K, Williams B, Bertoncello I. Evidence of an epithelial stem/progenitor cell hierarchy in the adult mouse lung. Proc Natl Acad Sci US A. 2010;107(4):1414-1419.

9. Fehrenbach H. Alveolar epithelial type II cell: defender of the alveolus revisited. Respir Res. 2001;2(1):33-46.

10. Thannickal VJ, Toews GB, White ES, Lynch JP 3rd, Martinez FJ. Mechanisms of pulmonary fibrosis.
Annu Rev Med. 2004;55:395-417.

11. Raymond K, Kreft M, Janssen H, Calafat J, Sonnenberg A. Keratinocytes display normal proliferation, survival and differentiation in conditional beta4-integrin knockout mice. J Cell Sci. 2005; 118(pt 5):1045-1060.

12. Kim KK, et al. Epithelial cell alpha3beta 1 integrin links beta-catenin and Smad signaling to promote myofibroblast formation and pulmonary fibrosis. J Clin Invest. 2009;119(1):213-224.

13. Kim N, Yamamoto H, Pauling MH, Lorizio W, Vu $\mathrm{TH}$. Ablation of lung epithelial cells deregulates FGF10 expression and impairs lung branching morphogenesis. Anat Rec (Hoboken). 2009;292(1):123-130.

14. Giangreco A, Reynolds SD, Stripp BR. Terminal bronchioles harbor a unique airway stem cell population that localizes to the bronchoalveolar duct junction. Am J Pathol. 2002;161(1):173-182.

15. Kim CF, et al. Identification of bronchioalveolar stem cells in normal lung and lung cancer. Cell. 2005; 121(6):823-835.

16. Reddy $\mathrm{R}$, et al. Isolation of a putative progenitor subpopulation of alveolar epithelial type 2 cells. $A m J$ Physiol Lung Cell Mol Physiol. 2004;286(4):L658-L667.

17. Zimmermann B. Lung organoid culture. Differentiation. 1987;36(1):86-109.

18. Rawlins EL, Ostrowski LE, Randell SH, Hogan BL. Lung development and repair: contribution of the ciliated lineage. Proc Natl Acad Sci U S A. 2007; 104(2):410-417.

19. Wuenschell CW, Sunday ME, Singh G, Minoo P, Slavkin HC, Warburton D. Embryonic mouse lung epithelial progenitor cells co-express immunohistochemical markers of diverse mature cell lineages. J Histochem Cytochem. 1996;44(2):113-123.

20. Szymczak AL, Vignali DA. Development of 2A peptide-based strategies in the design of multicistronic vectors. Expert Opin Biol Ther. 2005;5(5):627-638.

21. Snyder JC, Teisanu RM, Stripp BR. Endogenous lung stem cells and contribution to disease.J Pathol. 2009;217(2):254-264.

22. Rawlins EL, et al. The role of Scgb1a1+ Clara cells in the long-term maintenance and repair of lung airway, but not alveolar, epithelium. Cell Stem Cell. 2009;4(6):525-534.

23. Adamson IY, Bowden DH. The type 2 cell as progenitor of alveolar epithelial regeneration. A cytodynamic study in mice after exposure to oxygen. Lab Invest. 1974;30(1):35-42.

24. Raymond K, Kreft M, Song JY, Janssen H, Sonnenberg A. Dual Role of alpha6beta4 integrin in epidermal tumor growth: tumor-suppressive versus tumor-promoting function. Mol Biol Cell. 2007;18(11):4210-4221

25. Mani SA, et al. The epithelial-mesenchymal transition generates cells with properties of stem cells. Cell. 2008;133(4):704-715.

26. Kim KK, et al. Alveolar epithelial cell mesenchymal transition develops in vivo during pulmonary fibrosis and is regulated by the extracellular matrix. Proc Natl Acad Sci U S A. 2006;103(35):13180-13185.

27. Teisanu RM, Lagasse E, Whitesides JF, Stripp BR. Prospective isolation of bronchiolar stem cells based upon immunophenotypic and autofluorescence characteristics. Stem Cells. 2009;27(3):612-622.

28. Rock JR, et al. Basal cells as stem cells of the mouse trachea and human airway epithelium. Proc Natl Acad Sci U S A. 2009;106(31):12771-12775.

29. Hayward SW, et al. Interactions between adult human prostatic epithelium and rat urogenital sinus mesenchyme in a tissue recombination model. Differentiation. 1998;63(3):131-140. 